\title{
Cooling and entangling ultracold atoms in optical lattices
}

\author{
Bing Yang, ${ }^{1,2,3}$, Hui Sun ${ }^{1,2,3}$, Chun-Jiong Huang ${ }^{1,3}$, Han-Yi Wang, ${ }^{1,2,3}$, Youjin Deng1,3, Han-Ning Dai ${ }^{1,2,3}$, \\ Zhen-Sheng Yuan ${ }^{1,2,3 *}$, Jian-Wei Pan ${ }^{1,2,3 *}$
}

${ }^{1}$ Hefei National Laboratory for Physical Sciences at Microscale and Department of Modern Physics, University of Science and Technology of China, Hefei, Anhui 230026, China. ${ }^{2}$ Physikalisches Institut, Ruprecht-Karls-Universität Heidelberg, 69120 Heidelberg, Germany. ${ }^{3} \mathrm{CAS}$ Centre for Excellence in Quantum Information and Quantum Physics, University of Science and Technology of China, Hefei, Anhui 230026, China.

*Corresponding author. Email: yuanzs@ustc.edu.cn (Z.-S.Y.); pan@ustc.edu.cn (J.-W.P.)

Scalable, coherent many-body systems can enable the realization of previously unexplored quantum phases and have a potential to exponentially speed up information processing. Here we report the cooling of a quantum simulator with ten thousand atoms and mass production of high-fidelity entangled pairs. In a two-dimensional plane, we cool Mott-insulator samples by immersing them into removable superfluid reservoirs, achieving an entropy per particle of $1.9_{-0.4}^{+1.7} \times 10^{-3} \mathrm{k}_{\mathrm{B}}$. The atoms are then rearranged into a twodimensional lattice free of defects. We further demonstrate a two-qubit gate with a fidelity of 0.993(1) for entangling 1250 atom pairs. Our results offer a setting for exploring low-energy many-body phases and could enable the creation of large-scale entanglement.

A paradigmatic quantum simulator is composed of ultracold neutral atoms in an optical lattice, which provides a clean and controllable platform for studying complex many-body problems $(1,2)$. However, the thermal entropy arising from the intrinsic heating $(3,4)$, non-adiabaticity $(5,6)$ and inefficient thermalization (7) hinders the exploration of exotic phases of matter (8) and scaling up entangled states $(9,10)$. Numerous methods have been applied to cool quantum gases (11-16); some theoretical schemes suggest immersing lattice trapped atoms into superfluid reservoirs that could eventually carry away the thermal entropy $(17,18)$. With the help of a microscope, cooling an atomic sample with a removable surrounding reservoir was demonstrated, leading to the realization of a Fermi-Hubbard antiferromagnet (5, 19). However, to further cool the atomic samples, either mixing two distinct quantum phases for efficient thermalization or realizing sub-lattice addressability to remove the reservoir is required, posing outstanding experimental challenges.

Here we design a staggered lattice to immerse samples into superfluid reservoirs for sufficient thermalization. Meanwhile, employing a sub-lattice addressing technique, all the high-entropy reservoirs are removed and a lowentropy regime is achieved. The cooling concept is sketched in Fig. 1A, where a superfluid that holds a large density of states serves as a reservoir for storing the entropy of the joint system. In our 2D bosonic system with a bichromatic superlattice along the $x$ direction, the competition between the kinetic energy $J$ and interaction energy $U$ leads to the formation of superfluid and Mott insulator phases. A spa- tially alternating appearance of the insulator and superfluid is realized by adjusting the local chemical potentials. Based on the local density variations, we develop a thermometry method to characterize the cooling performance. After isolating and removing the particles in the reservoirs, the atoms of the samples (Fig. 1B) are redistributed within the superlattice to achieve a unity-filling state with over $10^{4}$ sites.

The defect-free platform after cooling is a good starting point for quantum information processing. Owing to the parallel controllability of atoms in optical lattices, multipartite entanglement can be created by coupling the atomic qubits with nearest-neighbor interactions $(20,21)$. Although the single- and two-qubit control (22-26) have been demonstrated in this system, the current challenge toward achieving large-scale entanglement is to develop a high-fidelity entangling gate $(27,28)$. Here we enhance the superexchange interaction by engineering the Hamiltonian in a three-level scheme (Fig. 1C). The dynamical evolution is driven by the coherent competition of superexchange and atom tunnelling. Compared with an earlier experiment (26), we dramatically reduce the gate operation time and thereby significantly improve the operation fidelity, exceeding the threshold of some fault-tolerant models for quantum computing (29-31).

The experiment starts with a ${ }^{87} \mathrm{Rb}$ Bose-Einstein condensate of $\sim 8.6 \times 10^{4}$ atoms confined in a single well of a pancake-shaped standing wave (26). The $2 \mathrm{D}$ cloud is adiabatically loaded into a square lattice in the $x-y$ plane with a period of $\lambda_{\mathrm{s}} / 2$, where $\lambda_{\mathrm{s}}=767 \mathrm{~nm}$ denotes the wavelength of 
the "short" lattices. Another "long" lattice with wavelength $\lambda_{1}=2 \lambda_{\mathrm{s}}$ is employed to construct a superlattice that divides the quantum gas into odd and even subsystems. Their lattice depths are ramped exponentially in $60 \mathrm{~ms}$ with a time constant of $20 \mathrm{~ms}$ up to $V_{\mathrm{s}}=26.1(2) E_{\mathrm{r}}$ and $V_{1}=10.0(1) E_{\mathrm{r}}$, where $E_{\mathrm{r}}=h^{2} /\left(2 m \lambda_{\mathrm{s}}^{2}\right)$ is the recoil energy with $h$ the Planck constant and $m$ the atomic mass. Meanwhile, the transverse trap is adjusted to set the atomic densities to $\bar{n} \approx 1.75$ in the central area. The energy offset $\Delta$ between odd and even subsystems is controlled by shifting the relative phase of the superlattice lasers (26). As the tunneling strength decreases across a critical value, gapped Mott insulators with $\bar{n}=2$ start to separate from the adjacent superfluid states with $\bar{n}$ $\approx 1.5$.

After the atoms enter the deep lattices, coherent tunneling is negligible and defects in Mott insulators are induced only by thermal fluctuations (13). The on-site number fluctuations are probed via photoassociation collisions, which reduce the occupation of a site to its odd-even parity. The odd (sample) and even (reservoir) subsystems are distinguished by turning on a spin-dependent superlattice to separate their hyperfine transition by $28 \mathrm{kHz}(26)$. Then, we perform a Landau-Zener sweep to transfer the atoms of the even rows from the initial state $|\downarrow\rangle \equiv\left|F=1, m_{F}=-1\right\rangle$ to $|\uparrow\rangle \equiv$ $\left|F=2, m_{F}=-2\right\rangle$, with an efficiency of $99.5(3) \%$ (32). The in situ atomic densities $\bar{n}_{\text {det }}$ of the odd and even subsystems (Fig. 2A) are successively recorded by absorption imaging with an optical resolution of $\sim 1.0 \mu \mathrm{m}$. Figure $2 \mathrm{~B}$ shows the density profiles, where the number fluctuations of the samples decrease dramatically compared to the Mott insulator without applying the staggered-immersion cooling. Nevertheless, the efficiency of the parity projection $98.7(3) \%$ leaves undesired residual atoms, which limits the lowest entropy that can be detected to $0.011(2) k_{\mathrm{B}}$. To overcome this imperfection for determining the ultra-low temperature $T_{\mathrm{f}}$ of the target samples $(\Delta=-U / 2)$, we apply thermometry on the samples via probing the density redistribution in the odd/even subsystems as described below.

The partitions of atomic densities are measured at different offsets of the local chemical potential. Figure $2 \mathrm{C}$ shows the densities at different $\Delta / U$, where the Mott insulators emerge associated with superfluid states in the other subsystem. Changing $\Delta$ represents a small perturbation to the lattice, thus the size of the whole cloud is maintained and only local transport is allowed. Eight plateaus emerge around the half-integers of $\Delta / U$, which are analogous to the concentric shell structures of the Mott insulator in a harmonic trap (13). Intriguingly, the compressible superfluid states exhibit distinctive half-integer plateaus owing to the local particle conservation. In the absence of global mass flow, the entropy is most likely to be conserved locally and the nonadiabaticities of the lattice loading are minimized (6). In addition, we measure the thermalization rate by monitoring the quench dynamics (following a reversal of the sign of $\Delta$ at certain times during the lattice ramping), and find that the system can equilibrate on the time scale of $h / J$ (fig. S5) (32).

The temperature of the samples is fitted based on particle and entropy conservation at different $\Delta / U$. Figure $3 \mathrm{~A}$ shows $\bar{n}_{\text {det }}$ of the odd subsystem as a function of $\Delta / U$ at two experimental settings. The phase transition at the edge of the plateaus leads to sudden changes of the occupancies, which allows the thermometer to access ultra-low temperatures. In these samples, the lowest temperature achieved is $k_{\mathrm{B}} T_{\mathrm{f}}=0.046(10) U$. To characterize the cooling performance, we control the entropy by changing the adiabaticity or duration of the lattice loading $(3,6)$. For each superlattice loading sequence, a Mott insulator with central density $\bar{n}=2$ is prepared by setting $\Delta=0$ to disable the cooling effect. Thereby the entropy in the absence of cooling is deduced from the parity measurement. As shown in Fig. 3B, if the entropy without cooling achieves a critical value $\mathcal{S} / N$ $0.17 k_{\mathrm{B}}$, the cooling power becomes strong and the entropy of the samples reduces significantly. This corresponds to a phase transition of the reservoir from a normal fluid to a superfluid (4), achieving an entropy per particle of below $k_{\mathrm{B}} \ln 2 /(2 \bar{n})=0.20 k_{\mathrm{B}}$ as the system equilibrates at nonzero tunneling strength (32). The experimental results are in excellent agreement with the quantum Monte-Carlo simulations (32). In addition, the temperatures of smaller samples can be determined from the density profiles, and are consistent with the thermometry method above. Losing less than half of the total atoms in the reservoir ( 1.5/3.5), we lower the entropy per particle in the large samples to $\mathcal{S} / N=$ $1.9_{-0.4}^{+1.7} \times 10^{-3} k_{\mathrm{B}}$, corresponding to a 65 -fold reduction of the original entropy. Besides the entropy inherent in the $2 \mathrm{D}$ gas, the intrinsic heating induced by the light-induced scattering during the lattice loading process is $0.025(2) k_{\mathrm{B}}$ per particle (32), indicating that the cooling power has exceeded the heating rate of the optical lattices.

We implement state engineering over $10^{4}$ sites to turn the low-entropy samples into a unity-filling state. This is achieved by manipulating the double wells (DWs) in parallel after removing the particles in the high-entropy reservoirs. Figure 1B shows the sequence from the number state $|2,0\rangle$ to $|1,1\rangle$ in an isolated DW unit. To avoid level crossing causing band excitation, we shift the centroid of atom pairs to match the antinodes of the long-lattice before transferring the atoms adiabatically into its ground band. Next, we ramp up the short-lattice to split the atom pairs into a product state with one atom per site. The atoms favor occupying 
different sites in the presence of the interaction blockade $U$. The achieved fidelity of $99.3(1) \%$ is calibrated by applying multiple operations to the samples, i.e., driving the atoms between $|2,0\rangle$ and $|1,1\rangle$ back and forth as in Fig. 3C. Together with the entropy from the samples, the overall fidelity for preparing a unity filling state over $10^{4}$ lattice sites is 99.2(1)\%.

The defect-free platform provides massive physical qubits for quantum information processing (26-28). We implement the entangling gate within all the DWs using a three-level scheme. For probing the spin dynamics, the state initialization and detection have the same procedure as our previous experiment (26). Here, the potentials are set to 9.76(1) $E_{\mathrm{r}}$ for short-lattice and 10.0(1) $E_{\mathrm{r}}$ for long-lattice. In Fig. 4B, we observe an interference between two processes for 6.5 superexchange periods, which is equivalent to the application of 26 gate operations. The lifetime $57(16) \mathrm{ms}$ of the spin dynamics (shown in Fig. 4B) is mainly caused by the spatial inhomogeneity of the interactions and fluctuations of the superlattice phase (26). We further study the decoherence caused by light scattering and achieve a coherent oscillation with a lifetime of 646(32) ms (fig. S9) (32).

The gate fidelity is characterized by measuring the target Bell state $|t\rangle$, which is acquired by performing a twoqubit gate followed by a phase rotation (32). The fidelity of the experimentally produced density matrix is

$$
F=\left\langle t\left|\rho^{\exp }\right| t\right\rangle=\frac{1}{2}\left(P_{|\uparrow, \downarrow\rangle}+P_{|\downarrow, \uparrow\rangle}\right)+\frac{1}{4}\left(\left\langle\sigma_{x} \sigma_{x}\right\rangle+\left\langle\sigma_{y} \sigma_{y}\right\rangle\right)
$$

where $\sigma_{x, y}$ are Pauli spin operators. The bases for the $\sigma_{x}$ and $\sigma_{y} \quad$ operators are $\left.\quad|+|-\right\rangle=(|\uparrow\rangle \pm|\downarrow\rangle) / \sqrt{2} \quad$ and $|\circlearrowright / \circlearrowleft\rangle=(|\uparrow\rangle \pm i|\downarrow\rangle) / \sqrt{2}$, respectively. The incoherent error of the gate operator accumulates and the fidelity scales as $F^{N}$ when multiple gates $N$ are applied. From the measurements of the state populations (Fig. 4C), we observe a reduction of the state coherence after implementing multiple quantum gates. To avoid the influence of the detection error, we measure the fidelity of the Bell state after 29 consecutive gate operations and then deduce the single-gate fidelity. Under the $|\uparrow / \downarrow\rangle$ basis, the probabilities of the states are $P_{|\uparrow, \downarrow\rangle}=0.44(2), P_{|\downarrow, \uparrow\rangle}=0.42(1), P_{|\uparrow, \uparrow\rangle}=0.01(2), P_{|\downarrow, \downarrow\rangle}=$ $0.01(3)$. The states $\{|\uparrow \downarrow, 0\rangle,|0, \uparrow \downarrow\rangle\}$ resulting from the operation error are included in the normalization. For the basis of $|+|-\rangle$ and $|\circlearrowright / \circlearrowleft\rangle$, a microwave $\pi / 2$ pulse is applied to the atoms and then the populations of the spin states are measured. The $\sigma_{y}$ operator given by the pulse has a relative $\pi / 2$ phase shift with the pulse of the $\sigma_{x}$ operator. The expectation values of the spin correlations are $\left\langle\sigma_{x} \sigma_{x}\right\rangle=0.75(3)$ and $\left\langle\sigma_{y} \sigma_{y}\right\rangle=0.75(4)$. The fidelity of this state after 29 gates is
0.80 (2), from which the fidelity of an individual two-qubit gate is deduced as $F=99.3(1) \%$. The infidelity of this twoqubit gate is below the error threshold required by some fault-tolerant models (29-31).

In conclusion, we have demonstrated a cooling method to dramatically reduce the entropy of a quantum simulator. The achieved entropy enables studies of low-energy physics. Furthermore, we have realized a high-fidelity two-qubit gate and prepared 1250 Bell states of neutral atoms in parallel. The defect-free atom arrays and entangling gate are essential building blocks for creating multi-particle entanglement toward measurement-based quantum computing $(28,33$, 34). For building a practical logic qubit toward faulttolerant quantum computation (29-31), our system meets the requirements both concerning the entangling gate and the number of physical qubits. Our technique is generic and practical for cooling insulating states of other species and systems in other geometries (18). For the gapless fermionic phases, our method could significantly improve the efficiency of entropy transport (12, 19), giving access to lowertemperature regimes for observing the $d$-wave superfluid phase (35). The defect-free platform and entangled pairs are suitable for exploring the bosonic antiferromagnetic order (36) and resonating valence bond states (37).

\section{REFERENCES AND NOTES}

1. I. Bloch, J. Dalibard, W. Zwerger, Many-body physics with ultracold gases. Rev. Mod. Phys. 80, 885-964 (2008). doi:10.1103/RevModPhys.80.885

2. M. Greiner, O. Mandel, T. Esslinger, T. W. Hänsch, I. Bloch, Quantum phase transition from a superfluid to a Mott insulator in a gas of ultracold atoms. Nature 415, 39-44 (2002). doi:10.1038/415039a Medline

3. H. Pichler, A. J. Daley, P. Zoller, Nonequilibrium dynamics of bosonic atoms in optical lattices: Decoherence of many-body states due to spontaneous emission. Phys. Rev. A 82, 063605 (2010). doi:10.1103/PhysRevA.82.063605

4. S. Trotzky, L. Pollet, F. Gerbier, U. Schnorrberger, I. Bloch, N. V. Prokof'ev, B. Svistunov, M. Troyer, Suppression of the critical temperature for superfluidity near the Mott transition. Nat. Phys. 6, 998-1004 (2010). do:10.1038/nphys1799

5. C. S. Chiu, G. Ji, A. Mazurenko, D. Greif, M. Greiner, Quantum State Engineering of a Hubbard System with Ultracold Fermions. Phys. Rev. Lett. 120, 243201 (2018). doi:10.1103/PhysRevLett.120.243201 Medline

6. M. Dolfi, A. Kantian, B. Bauer, M. Troyer, Minimizing nonadiabaticities in opticallattice loading. Phys. Rev. A 91, 033407 (2015). doi:10.1103/PhysReva.91.033407

7. C.-L. Hung, X. Zhang, N. Gemelke, C. Chin, Slow mass transport and statistical evolution of an atomic gas across the superfluid-Mott-insulator transition. Phys. Rev. Lett. 104, 160403 (2010). doi:10.1103/PhysRevLett.104.160403 Medline

8. D. C. McKay, B. DeMarco, Cooling in strongly correlated optical lattices: Prospects and challenges. Rep. Prog. Phys. 74, 054401 (2011). doi:10.1088/00344885/74/5/054401

9. M. Nielsen, I. L. Chuang, Quantum Computation and Quantum Information (Cambridge Univ. Press, 2010).

10. P. W. Shor, in 37th Symposium on Foundations of Computing (IEEE Computer Society Press, 1996), pp. 56-65.

11. J. Catani, G. Barontini, G. Lamporesi, F. Rabatti, G. Thalhammer, F. Minardi, S. Stringari, M. Inguscio, Entropy exchange in a mixture of ultracold atoms. Phys. 
Rev. Lett. 103, 140401 (2009). doi:10.1103/PhysRevLett.103.140401 Medline

12. T.-L. Ho, Q. Zhou, Universal Cooling Scheme for Quantum Simulation. arXiv 0911.5506 [cond-mat.quant-gas] (29 November 2009).

13. W. S. Bakr, A. Peng, M. E. Tai, R. Ma, J. Simon, J. I. Gillen, S. Fölling, L. Pollet, M. Greiner, Probing the superfluid-to-Mott insulator transition at the single-atom level. Science 329, 547-550 (2010). doi:10.1126/science.1192368 Medline

14. P. Medley, D. M. Weld, H. Miyake, D. E. Pritchard, W. Ketterle, Spin gradient demagnetization cooling of ultracold atoms. Phys. Rev. Lett. 106, 195301 (2011). doi:10.1103/PhysRevLett.106.195301 Medline

15. S. Taie, R. Yamazaki, S. Sugawa, Y. Takahashi, An SU(6) Mott insulator of an atomic Fermi gas realized by large-spin Pomeranchuk cooling. Nat. Phys. 8 825-830 (2012). doi:10.1038/nphys2430

16. D. Greif, T. Uehlinger, G. Jotzu, L. Tarruell, T. Esslinger, Short-range quantum magnetism of ultracold fermions in an optical lattice. Science 340, 1307-1310 (2013). doi:10.1126/science.1236362 Medline

17. A. Griessner, A. J. Daley, S. R. Clark, D. Jaksch, P. Zoller, Dark-state cooling of atoms by superfluid immersion. Phys. Rev. Lett. 97, 220403 (2006). doi:10.1103/PhysRevLett.97.220403 Medline

18. A. Kantian, S. Langer, A. J. Daley, Dynamical Disentangling and Cooling of Atoms in Bilayer Optical Lattices. Phys. Rev. Lett. 120, 060401 (2018). doi:10.1103/PhysRevLett.120.060401 Medline

19. A. Mazurenko, C. S. Chiu, G. Ji, M. F. Parsons, M. Kanász-Nagy, R. Schmidt, F. Grusdt, E. Demler, D. Greif, M. Greiner, A cold-atom Fermi-Hubbard antiferromagnet. Nature 545, 462-466 (2017). doi:10.1038/nature22362 Medline

20. D. Jaksch, H.-J. Briegel, J. I. Cirac, C. W. Gardiner, P. Zoller, Entanglement of Atoms via Cold Controlled Collisions. Phys. Rev. Lett. 82, 1975-1978 (1999). doi:10.1103/PhysRevLett.82.1975

21. L. M. Duan, E. Demler, M. D. Lukin, Controlling spin exchange interactions of ultracold atoms in optical lattices. Phys. Rev. Lett. 91, 090402 (2003). doi:10.1103/PhysRevLett.91.090402 Medline

22. C. Weitenberg, M. Endres, J. F. Sherson, M. Cheneau, P. Schauss, T. Fukuhara, I. Bloch, S. Kuhr, Single-spin addressing in an atomic Mott insulator. Nature 471, 319-324 (2011). doi:10.1038/nature09827 Medline

23. Y. Wang, A. Kumar, T.-Y. Wu, D. S. Weiss, Single-qubit gates based on targeted phase shifts in a 3D neutral atom array. Science 352, 1562-1565 (2016). doi:10.1126/science.aaf2581 Medline

24. M. Anderlini, P. J. Lee, B. L. Brown, J. Sebby-Strabley, W. D. Phillips, J. V. Porto, Controlled exchange interaction between pairs of neutral atoms in an optical lattice. Nature 448, 452-456 (2007). doi:10.1038/nature06011 Medline

25. S. Trotzky, P. Cheinet, S. Fölling, M. Feld, U. Schnorrberger, A. M. Rey, A. Polkovnikov, E. A. Demler, M. D. Lukin, I. Bloch, Time-resolved observation and control of superexchange interactions with ultracold atoms in optical lattices. Science 319, 295-299 (2008). doi:10.1126/science.1150841 Medline

26. H.-N. Dai, B. Yang, A. Reingruber, X.-F. Xu, X. Jiang, Y.-A. Chen, Z.-S. Yuan, J.-W. Pan, Generation and detection of atomic spin entanglement in optical lattices. Nat. Phys. 12, 783-787 (2016). doi:10.1038/nphys3705

27. T. D. Ladd, F. Jelezko, R. Laflamme, Y. Nakamura, C. Monroe, J. L. O'Brien, Quantum computers. Nature 464, 45-53 (2010). doi:10.1038/nature08812 Medline

28. R. Raussendorf, H. J. Briegel, A one-way quantum computer. Phys. Rev. Lett. 86, 5188-5191 (2001). doi:10.1103/PhysRevLett.86.5188 Medline

29. E. Knill, Quantum computing with realistically noisy devices. Nature $434,39-44$ (2005). doi:10.1038/nature03350 Medline

30. R. Raussendorf, J. Harrington, Fault-tolerant quantum computation with high threshold in two dimensions. Phys. Rev. Lett. 98, 190504 (2007). doi:10.1103/PhysRevLett.98.190504 Medline

31. A. G. Fowler, M. Mariantoni, J. M. Martinis, A. N. Cleland, Surface codes: Towards practical large-scale quantum computation. Phys. Rev. A 86, 032324 (2012).
doi:10.1103/PhysRevA.86.032324

32. See supplementary materials.

33. R. Raussendorf, D. E. Browne, H. J. Briegel, Measurement-based quantum computation on cluster states. Phys. Rev. A 68, 022312 (2003). doi:10.1103/PhysRevA.68.022312

34. B. Vaucher, A. Nunnenkamp, D. Jaksch, Creation of resilient entangled states and a resource for measurement-based quantum computation with optical superlattices. New J. Phys. 10, 023005 (2008). doi:10.1088/13672630/10/2/023005

35. W. Hofstetter, J. I. Cirac, P. Zoller, E. Demler, M. D. Lukin, High-temperature superfluidity of fermionic atoms in optical lattices. Phys. Rev. Lett. 89, 220407 (2002). doi:10.1103/PhysRevLett.89.220407 Medline

36. A. S. Sørensen, E. Altman, M. Gullans, J. V. Porto, M. D. Lukin, E. Demler, Adiabatic preparation of many-body states in optical lattices. Phys. Rev. A 81 061603 (2010). doi:10.1103/PhysRevA.81.061603

37. S. Trebst, U. Schollwöck, M. Troyer, P. Zoller, d-Wave resonating valence bond states of fermionic atoms in optical lattices. Phys. Rev. Lett. 96, 250402 (2006). doi:10.1103/PhysRevLett.96.250402 Medline

38. B. Yang, Harvard Dataverse, doi.org/10.7910/DVN/XSFBAB.

39. B. Yang, H.-N. Dai, H. Sun, A. Reingruber, Z.-S. Yuan, J.-W. Pan, Spin-dependent optical superlattice. Phys. Rev. A 96, 011602 (2017). doi:10.1103/PhysRevA.96.011602

40. K. M. Jones, E. Tiesinga, P. D. Lett, P. S. Julienne, Ultracold photoassociation spectroscopy: Long-range molecules and atomic scattering. Rev. Mod. Phys. 78, 483-535 (2006). doi:10.1103/RevModPhys.78.483

41. A. Fioretti, C. Amiot, C. M. Dion, O. Dulieu, M. Mazzoni, G. Smirne, C. Gabbanini, Cold rubidium molecule formation through photoassociation: A spectroscopic study of the 0g- long-range state of 87Rb 2. Eur. Phys. J. D 15, 189-198 (2001). doi:10.1007/s100530170165

42. F. Gerbier, Boson Mott insulators at finite temperatures. Phys. Rev. Lett. 99, 120405 (2007). doi:10.1103/PhysRevLett.99.120405 Medline

43. T.-L. Ho, Q. Zhou, Intrinsic heating and cooling in adiabatic processes for bosons in optical lattices. Phys. Rev. Lett. 99, 120404 (2007). doi:10.1103/PhysRevLett.99.120404 Medline

44. D. Jaksch, C. Bruder, J. I. Cirac, C. W. Gardiner, P. Zoller, Cold Bosonic Atoms in Optical Lattices. Phys. Rev. Lett. 81, 3108-3111 (1998). doi:10.1103/PhysRevLett.81.3108

45. N. V. Prokof'ev, B. V. Svistunov, I. S. Tupitsyn, “Worm” algorithm in quantum Monte Carlo simulations. Phys. Lett. A 238, 253-257 (1998). doi:10.1016/S0375-9601(97)00957-2

46. N. V. Prokof'ev, B. V. Svistunov, I. S. Tupitsyn, Exact, complete, and universal continuous-time worldline Monte Carlo approach to the statistics of discrete quantum systems. J. Exp. Theor. Phys. 87, 310-321 (1998). doi:10.1134/1.558661

47. B. Capogrosso-Sansone, Ş. G. Söyler, N. Prokof'ev, B. Svistunov, Monte Carlo study of the two-dimensional Bose-Hubbard model. Phys. Rev. A 77, 015602 (2008). doi:10.1103/PhysRevA.77.015602

48. N. Teichmann, D. Hinrichs, M. Holthaus, A. Eckardt, Bose-Hubbard phase diagram with arbitrary integer filling. Phys. Rev. B 79, 100503 (2009). doi:10.1103/PhysRevB.79.100503

49. D. C. McKay, C. Meldgin, D. Chen, B. DeMarco, Slow thermalization between a lattice and free Bose gas. Phys. Rev. Lett. 111, 063002 (2013). doi:10.1103/PhysRevLett.111.063002 Medline

50. J. F. Sherson, C. Weitenberg, M. Endres, M. Cheneau, I. Bloch, S. Kuhr, Singleatom-resolved fluorescence imaging of an atomic Mott insulator. Nature 467 68-72 (2010). doi:10.1038/nature09378 Medline

51. P. Rabl, A. J. Daley, P. O. Fedichev, J. I. Cirac, P. Zoller, Defect-suppressed atomic crystals in an optical lattice. Phys. Rev. Lett. 91, 110403 (2003). doi:10.1103/PhysRevLett.91.110403 Medline 
52. M. Popp, J.-J. Garcia-Ripoll, K. G. Vollbrecht, J. I. Cirac, Ground-state cooling of atoms in optical lattices. Phys. Rev. A 74, 013622 (2006). doi:10.1103/PhysRevA.74.013622

53. P. Cheinet, S. Trotzky, M. Feld, U. Schnorrberger, M. Moreno-Cardoner, S. Fölling, I. Bloch, Counting atoms using interaction blockade in an optical superlattice. Phys. Rev. Lett. 101, 090404 (2008). doi:10.1103/PhysRevLett.101.090404 Medline

54. W. S. Bakr, P. M. Preiss, M. E. Tai, R. Ma, J. Simon, M. Greiner, Orbital excitation blockade and algorithmic cooling in quantum gases. Nature 480, 500-503 (2011). doi:10.1038/nature10668 Medline

55. M. C. Tichy, K. Mølmer, J. F. Sherson, Shaking the entropy out of a lattice: Atomic filtering by vibrational excitations. Phys. Rev. A 86, 033618 (2012). doi:10.1103/PhysRevA.86.033618

56. O. Mandel, M. Greiner, A. Widera, T. Rom, T. W. Hänsch, I. Bloch, Controlled collisions for multi-particle entanglement of optically trapped atoms. Nature 425, 937-940 (2003). doi:10.1038/nature02008 Medline

57. D. Jaksch, J. I. Cirac, P. Zoller, S. L. Rolston, R. Côté, M. D. Lukin, Fast quantum gates for neutral atoms. Phys. Rev. Lett. 85, 2208-2211 (2000). doi:10.1103/PhysRevLett.85.2208 Medline

58. L. Isenhower, E. Urban, X. L. Zhang, A. T. Gill, T. Henage, T. A. Johnson, T. G. Walker, M. Saffman, Demonstration of a neutral atom controlled-NOT quantum gate. Phys. Rev. Lett. 104, 010503 (2010). doi:10.1103/PhysRevLett.104.010503 Medline

59. H. Levine, A. Keesling, A. Omran, H. Bernien, S. Schwartz, A. S. Zibrov, M. Endres, M. Greiner, V. Vuletić, M. D. Lukin, High-Fidelity Control and Entanglement of Rydberg-Atom Qubits. Phys. Rev. Lett. 121, 123603 (2018). doi:10.1103/PhysRevLett.121.123603 Medline

60. A. Omran, H. Levine, A. Keesling, G. Semeghini, T. T. Wang, S. Ebadi, H. Bernien, A. S. Zibrov, H. Pichler, S. Choi, J. Cui, M. Rossignolo, P. Rembold, S. Montangero, T. Calarco, M. Endres, M. Greiner, V. Vuletić, M. D. Lukin, Generation and manipulation of Schrödinger cat states in Rydberg atom arrays. Science 365, 570-574 (2019). doi:10.1126/science.aax9743 Medline

61. R. Grimm, M. Weidemüller, Y. B. Ovchinnikov, Optical Dipole Traps for Neutral Atoms. Adv. At. Mol. Opt. Phys. 42, 95-170 (2000). doi:10.1016/S1049$250 \times(08) 60186-X$

62. D. J. Wineland, C. Monroe, W. M. Itano, D. Leibfried, B. E. King, D. M. Meekhof, Experimental issues in coherent quantum-state manipulation of trapped atomic ions. J. Res. Natl. Inst. Stand. Technol. 103, 259-328 (1998). doi:10.6028/ires.103.019 Medline

63. T. Lahaye, C. Menotti, L. Santos, M. Lewenstein, T. Pfau, The physics of dipolar bosonic quantum gases. Rep. Prog. Phys. 72, 126401 (2009). doi:10.1088/00344885/72/12/126401

64. C. Chin, R. Grimm, P. Julienne, E. Tiesinga, Feshbach resonances in ultracold gases. Rev. Mod. Phys. 82, 1225-1286 (2010). doi:10.1103/RevModPhys.82.1225

65. W. S. Bakr, J. I. Gillen, A. Peng, S. Fölling, M. Greiner, A quantum gas microscope for detecting single atoms in a Hubbard-regime optical lattice. Nature 462, 7477 (2009). doi:10.1038/nature08482 Medline

\section{ACKNOWLEDGMENTS}

We thank A. Kantian for discussions. Funding: This work has been supported by the National Key R\&D Program of China (Grant No. 2016YFA0301603), the NNSFC (No. 11874341), the Fundamental Research Funds for the Central Universities, Special funds for promoting the construction of world-class universities and disciplines, the Anhui Initiative in Quantum Information Technologies and the Chinese Academy of Sciences. Author contributions: Z.-S.Y. and J.-W.P. conceived the research and designed the experiment. B.Y. developed the cooling method. B.Y., H.S and H.-N.D. set up the experiment, B.Y., H.S. and H.-Y.W. performed the measurement and analyzed the data, C.-Y.H. and Y, D. did the quantum Monte-Carlo simulation. All authors contributed to manuscript preparation. Competing interests: The authors declare no competing financial interests. Data and materials availability: All experimental data and code are available in the database (38).

\section{SUPPLEMENTARY MATERIALS}

science.sciencemag.org/cgi/content/full/science.aaz6801/DC1

Supplementary Text

Figs. S1 to S10

Table S1

References (39-65)

30 September 2019; accepted 5 June 2020

Published online 18 June 2020

10.1126/science.aaz6801 


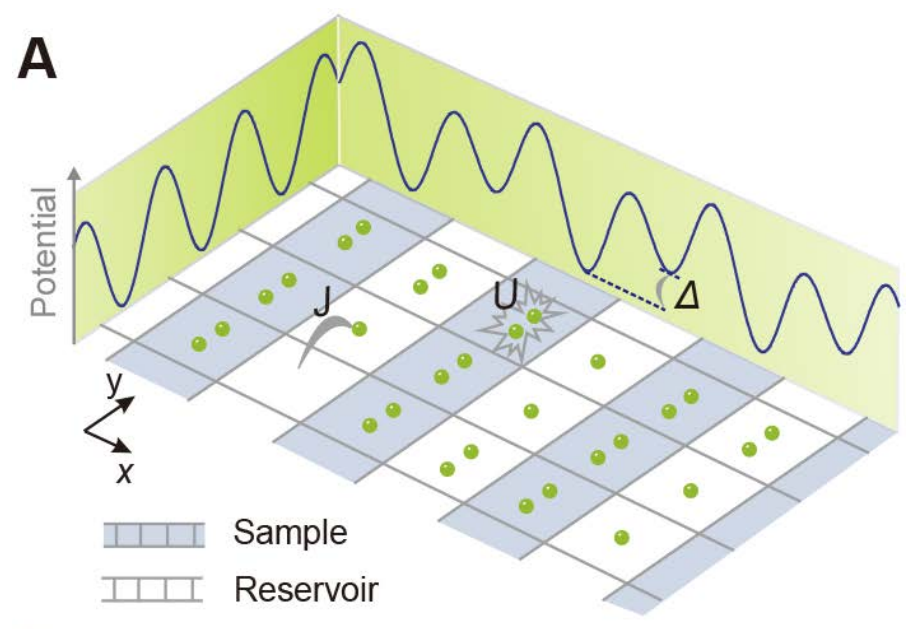

B
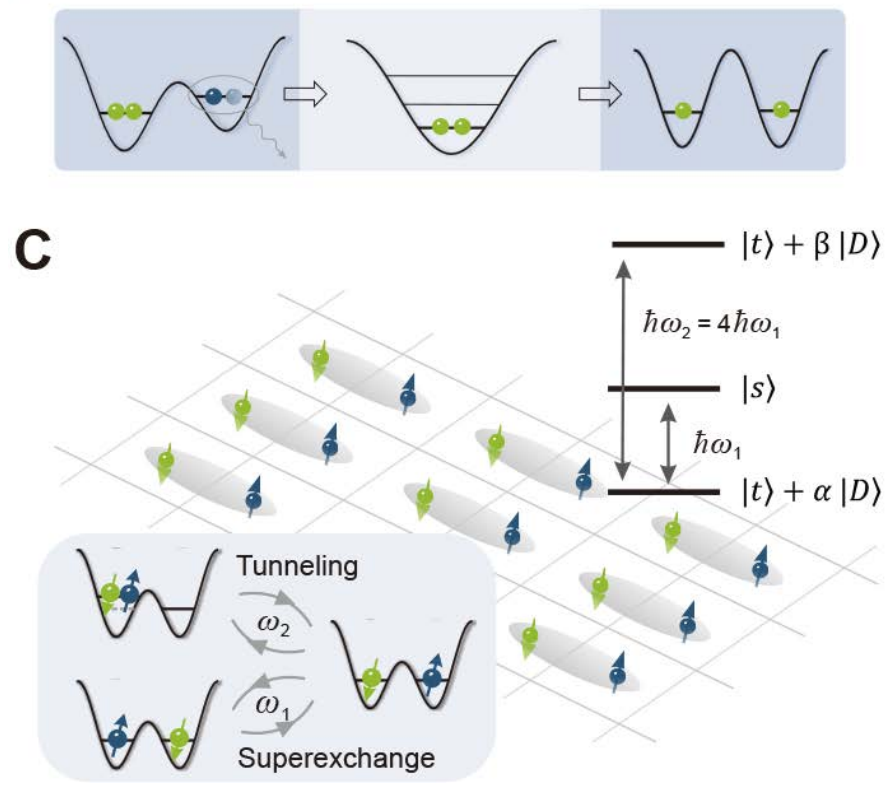

Fig. 1. Cooling and entangling atoms. (A) Schematic of staggered-immersion cooling. In an optical superlattice, gapped Mott insulators (samples) are immersed in the gapless superfluid reservoirs with a staggered geometry. (B) Redistribution of the atoms into a unity filling state by engineering the lattice potential. (C) Entangling thousands of atom pairs in parallel. The atoms rendered green (blue) are in the state of $|\downarrow\rangle(|\uparrow\rangle)$. The blue and light-blue atoms in (B) represent an average occupation of $\sim 1.5$ atoms in the state of $|\uparrow\rangle$. When the initial state in double-wells (DWs) is $|\downarrow, \uparrow\rangle$, the Hilbert space contains three eigenstates, see the energy level diagram. The triplet/singlet states are $|t / s\rangle=(|\downarrow, \uparrow\rangle \pm|\uparrow, \downarrow\rangle) / \sqrt{2}$, the doubleoccupied state is $|D\rangle=(|\uparrow \downarrow, 0\rangle+|0, \uparrow \downarrow\rangle) / \sqrt{2}$; the coefficients $\alpha$ and $\beta$ depend on $J$ and $U$. At $J / U=\sqrt{3} / 4$, the transition frequencies satisfy the relation $\omega_{2}=4 \omega_{1}$. A two-qubit $\sqrt{\text { SWAP gate }}$ is realized after the evolution time of $\pi /\left(2 \omega_{1}\right)$. 
A

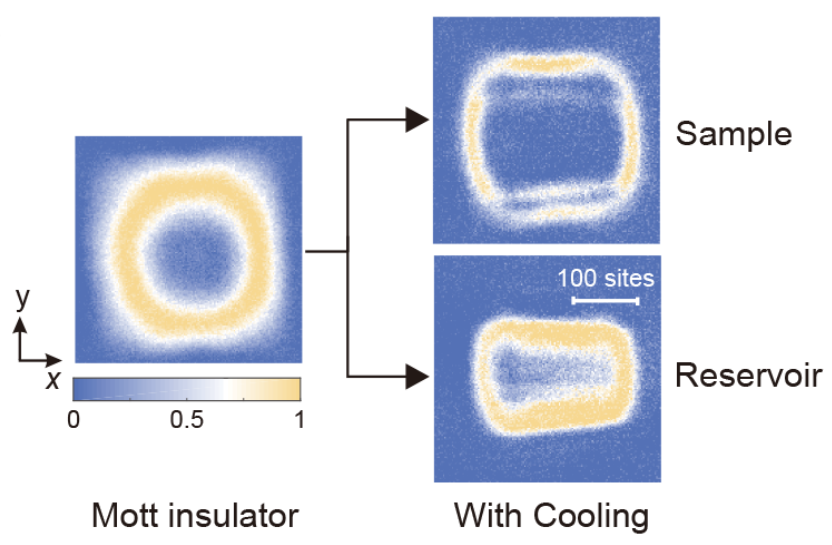

B

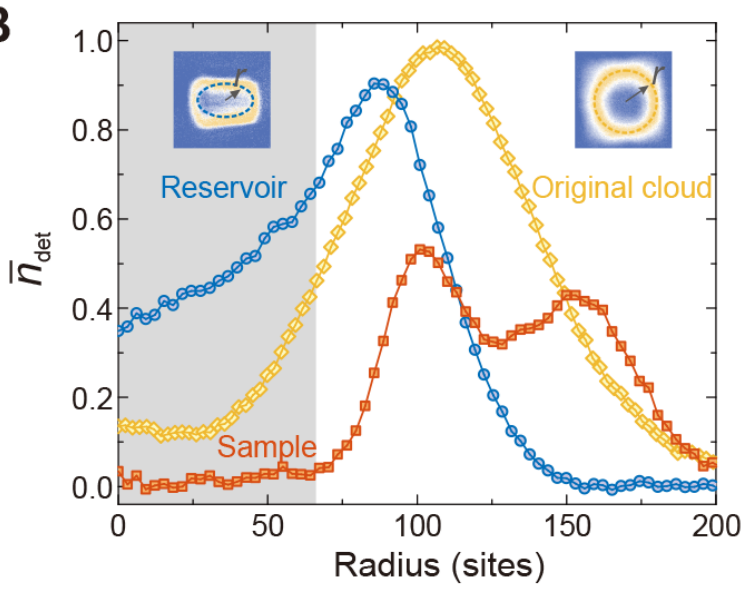

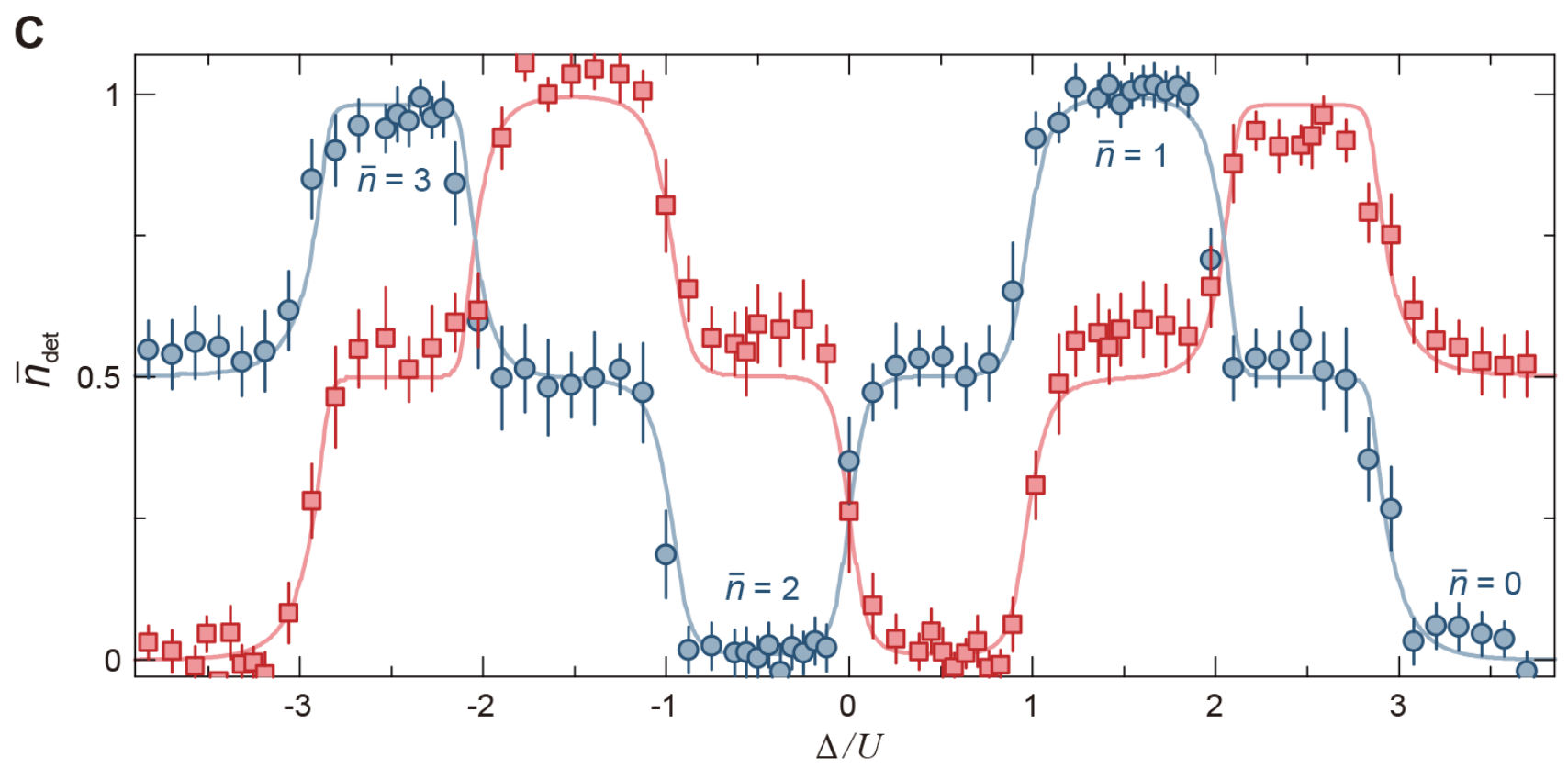

Fig. 2. Cooling a quantum gas. (A) Representative images of the atomic densities averaged over 50 repetitions. The original Mott insulator is created in the short-lattice without cooling. After applying the cooling sequence, we detect the odd (sample) and even (reservoir) subsystems separately. With a parityprojection measurement on the $\bar{n}=2$ Mott insulator, less remaining density in the cloud center indicates lower entropy. (B) The density profiles are azimuthal averages over the corresponding images in (A), as sketched in the insets. For calculating the mean densities in the samples (red) and the reservoirs (blue), radius $r$ refers to the geometric mean of the elliptical radii (the aspect ratio for this ellipse is 0.6). The entropy reduction is significant in the shaded area containing over $10^{4}$ lattice sites. (C) Density partitions between the odd (blue) and even (red) subsystems in the central region as a function of the energy offset between them. The superfluid and Mott insulator phases are coexistent and emerge alternately. Their densities change from 3.5 atoms per site to 0 and from 0 to 3.5 atoms per site, respectively, which is reflected in the parity-projection measurement. The staircase-like changing of the densities is indicative of the low temperature. The solid curves are isentropic simulations at $k_{\mathrm{B}} T_{\mathrm{f}} / U=0.05$ (fig. S4) (32). The subtle features, such as the larger statistical errors of the superfluid states, higher temperatures at larger $|\Delta|$ and unequal width of the Mott plateaus are revealed by our data and simulations. Each of the error bars represents the standard deviation over 20 images. 

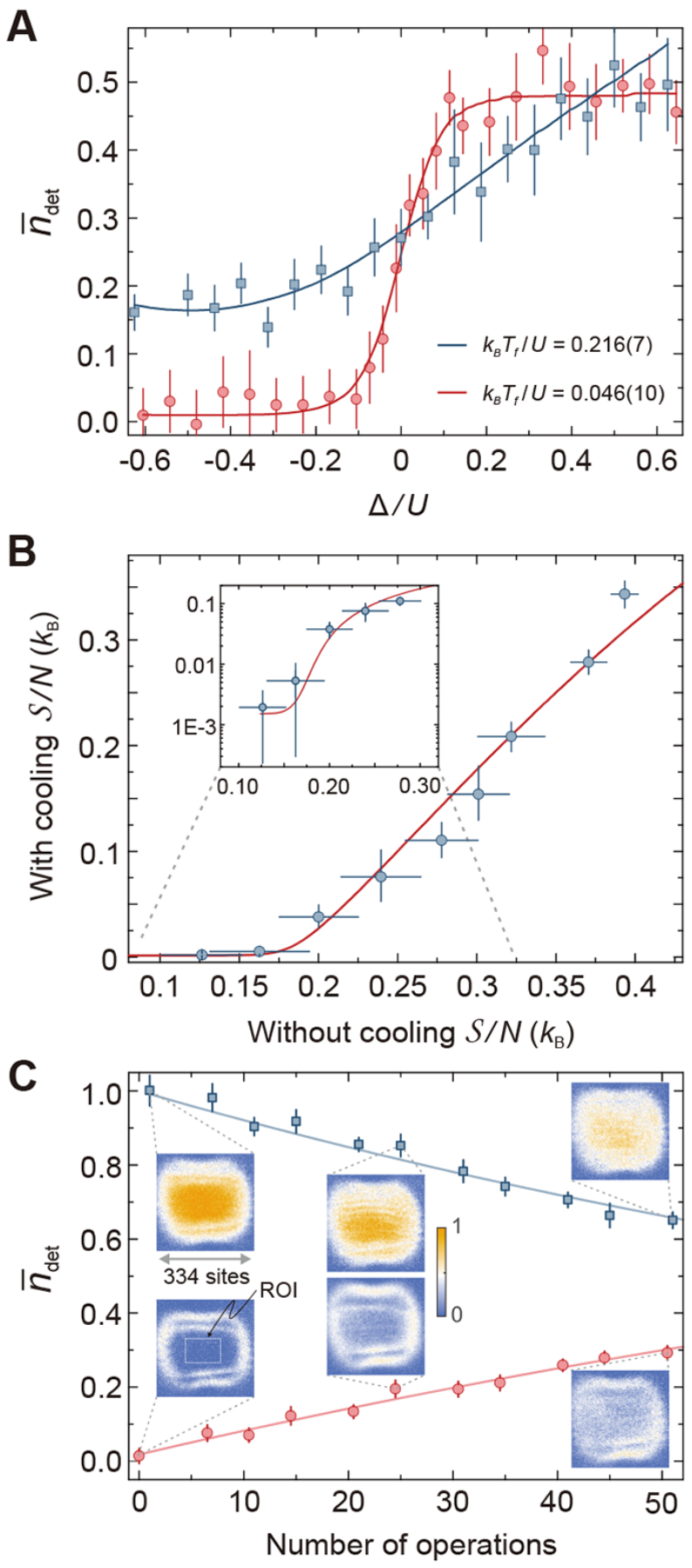

Fig. 3. Thermometry of the quantum gases and atom redistribution. (A) Density variations at $60 \mathrm{~ms}$ (red circles) and $500 \mathrm{~ms}$ (blue squares) lattice ramping. The corresponding curves are isentropic fitting results. (B) Cooling efficiency and the critical behavior. The red curve is the theoretical prediction based on Monte-Carlo simulations (fig. S4) (32). The inset shows the entropy of the system with cooling on a logarithmic scale. (C) Calibrating the fidelity of atom redistribution. We repeat the operation from $|2,0\rangle$ to $|1,1\rangle$ and its reversal in cycles, then count the atoms on $10^{4}$ lattice sites within the region of interest (ROI). Red circles (blue squares) correspond to parity-projection measurements after even (odd) times of the redistribution operation. Each data point is averaged over 20 measurements and the error bars denote the standard deviations. The red and blue curves are power-law fitting results. 

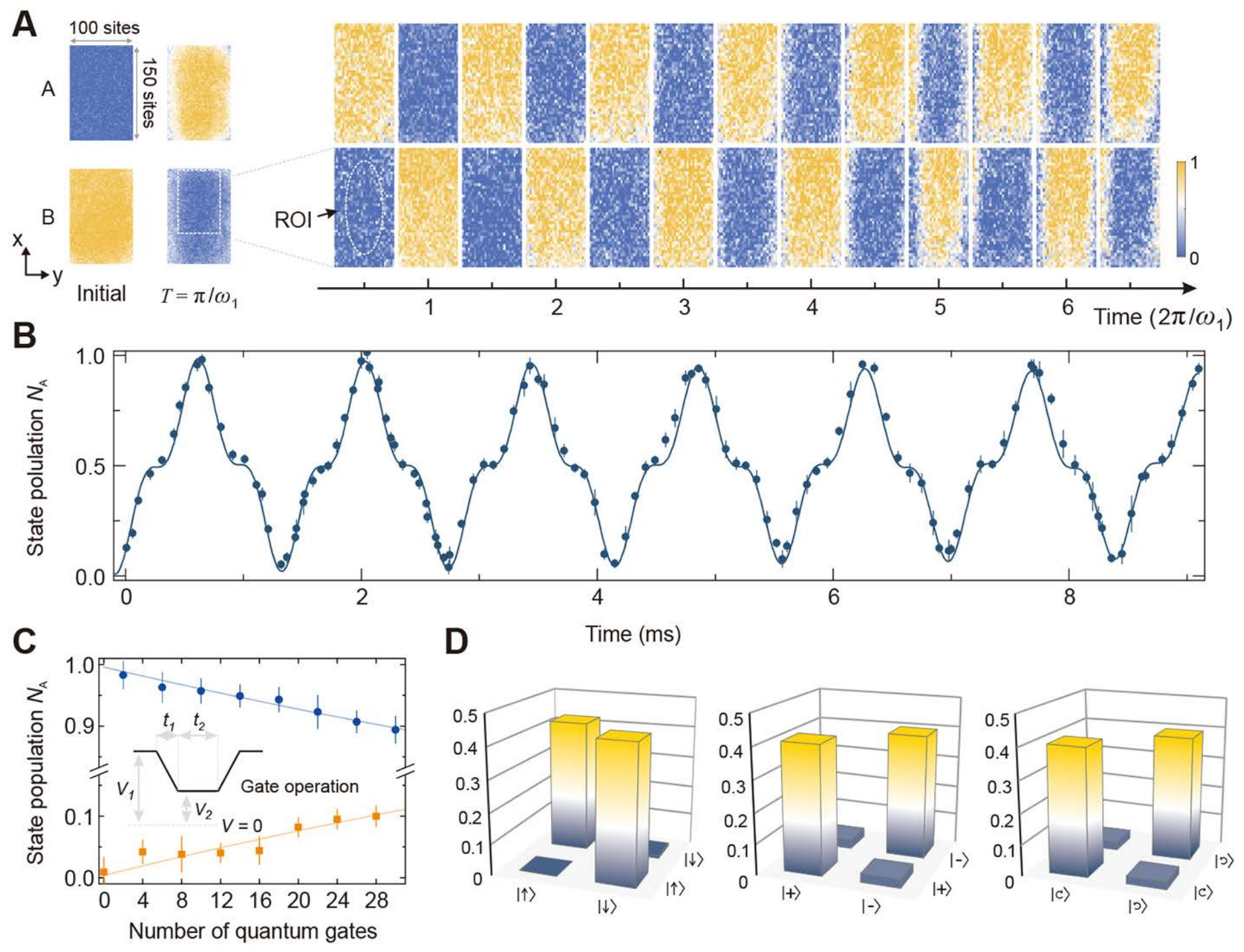

Fig. 4. Two-qubit quantum gate. (A) Spin dynamics. We measure the observables $N_{\mathrm{A}}=\langle n \uparrow$,odd $+n \downarrow$,even $\rangle / 2$ on image $A$ and $N_{B}=\langle n \downarrow$,odd $+n \uparrow$,even $\rangle / 2$ on image B, successively. The representative images show the initial state and the one after half superexchange period. The elliptical region of interest (ROI) containing 2500 lattice sites is chosen for counting signals. The high contrast of each groups of the images indicates excellent coherence of the system. The spatial inhomogeneity of the interactions among the sample starts to emerge after multi-periods. (B) Time-resolved observation of spin dynamics. The state evolution shows a coherent interference between two processes. Every data point is obtained by spatially averaging $N_{A}$ across the ROI and incorporating 5 experimental repetitions. The solid curve is a fitting result by considering the coherent evolution and a phenomenological exponential decay, which gives $\omega_{1} / 2 \pi=709(3) \mathrm{Hz}$ and a 57(16) ms lifetime. (C) Gate operation and state coherence. The quantum gate is implemented by controlling the depth of the short-lattice, where $V_{1}=31.2(2) E_{r}, V_{2}=9.72(7) E_{r}, t_{1}=250 \mu$ s and $t_{2}=270 \mu \mathrm{s}$. We apply multiple two-qubit gates and then record the state populations. Each data point is averaged over 20 measurements and the error bars are the standard deviations. The orange and blue symbols correspond to the measured $N_{A}$ after applying $4 j$ and $4 j+2$ gates respectively, with $j=0,1,2 \ldots 7$. The curves are power-law fitting results. (D) Spin correlations. The spin state after 29 gate operations, is measured on the $|\downarrow\rangle /|\uparrow\rangle,|+\rangle /|-\rangle$ and $|\circlearrowright\rangle /|\cup\rangle$ basis. 